\title{
The clinical relevance of lipohypertrophy
}

\section{S V Madhu ${ }^{1}$}

Published online: 3 August 2019

(C) Research Society for Study of Diabetes in India 2019
Lipohypertrophy (LH) has been a well recognized complication of insulin therapy for a long time. However, the true magnitude of its consequences for management of patients with diabetes is being realized lately. Several recent studies have not only shown that it is far more common than was traditionally believed, but also that its presence can significantly compromise the success of insulin therapy. Therefore, it becomes critical that all physicians and paramedical personnel involved in diabetes care should be alert in detecting and addressing the issue promptly and appropriately to ensure continued efficacy of insulin treatment.

It has been estimated that the prevalence of LH in insulintreated patients is $38 \%$ [1] based on a meta-analysis of 26 studies. Prevalence rates are higher among Asians compared with Europeans and also higher in type 2 diabetes mellitus patients (49\%) than in type 1 (34\%) [1]. The use of ultrasound has been shown by some to improve diagnostic sensitivity both in terms of the frequency and the extent of detection [2]. A recent audit from India [3] reported a much lower prevalence at $12.5 \%$ of all insulin users although this was a retrospective collection of cases based only on clinical examination and could be an underestimate while another survey [4] on injection technique found that based on a physical examination by nurses, one in five diabetic patients on insulin had LH. Two recent studies $[5,6]$ from India reported that more than $60 \%$ of the patients developed LH. Lipohypertrophy was found to be more prevalent in obese patients and those with hypoglycemia and higher HbA1C [7].

LH which appears as a rubbery swelling in the subcutaneous (SC) tissue at injecting sites is believed to develop secondary to the lipogenic action of insulin or to the trauma related to injections [5]. Various factors reported to be

S V Madhu

drsvmadhu@gmail.com

1 Department of Endocrinology, Centre for Diabetes, Endocrinology and Metabolism, University College of Medical Sciences \& GTB Hospital, Delhi, India associated with a greater risk of $\mathrm{LH}$ in diabetic patients include longer duration of insulin use, failure to injection site rotation, needle reuse [4], longer needles, longer duration of diabetes $[7,8]$, low BMI, total daily dose of insulin, twice daily insulin regimen, the use of conventional insulins compared with analogues [5], and poor glycemic control [8,9]. It has been shown that the risk of LH with insulin reuse is substantially higher than those who do not reuse. Seventy percent of those who reuse needles develop LH, and the risk has been shown to double when the needle has been reused 3-5 times [10].

In the current issue, Pahuja et al. [11] explore the frequency and factors associated with LH in a tertiary hospital from West India. The study confirms the high prevalence of LH (68\%) reported in recent studies from India. They report that longer duration of diabetes and longer duration of insulin use and the total daily dose of insulin were important risk factors. The study also highlights the gap between patient's awareness and management of LH reinforcing the need for increasing awareness about this important condition both among physicians and patients.

Several adverse consequences of LH on diabetes and its complications have been reported. Injection of insulin into areas of LH leads to its decreased and variable absorption that results in enhanced insulin requirements and glycemic variability, unexplained hypoglycemia, and more frequent diabetic ketoacidosis [4]. In fact, a sixfold higher occurrence of unexplained hypoglycemia and a sevenfold higher occurrence of glycemic variability have been reported in patients with $\mathrm{LH}$ compared with those without. The resultant poor glycemic control increases the risk of long-term diabetic complications such as cardiovascular disease, amputation, retinal diseases, and kidney disease [1]. Besides these, diabetic patients with LH require much higher doses of insulin leading to a greater financial burden.

Proper rotation of injection sites and avoiding needle reuse result in prevention or significant reduction of LH which has resulted in improved glycemic control [10]. In children with LH who received specific instructions from diabetic nurses to rotate injection sites and not to reuse needles, it was shown 
that in 3 months, $90 \%$ of LH lesions had resolved and glycemic control had significantly improved [4]. However, other workers reported no effect of avoiding reuse of needles [5]. Injection technique (IT) education, particularly with respect to use of shorter needles, has been shown to effectively prevent LH in prospective randomized controlled trials [12].

Achieving and sustaining good glycemic control in insulintreated patients is a major challenge and early detection of LH and timely correction of injection technique are critical in attaining this goal. The FIT, India guidelines endorsed by Research Society for Study of Diabetes in India as well as the Endocrine Society of India, provides useful guidance on best practices in this regard [13]. Clinicians involved in diabetes care would do well to make special efforts to focus on various aspects of insulin injection technique among insulin users for successful glycemic management in their practice.

\section{References}

1. Deng N, Zhang X, Zhao F, Wang Y, He H. Prevalence of lipohypertrophy in insulin-treated diabetes patients: a systematic review and meta-analysis. J Diabetes Investig. 2018;9(3):536-43.

2. Ghazaleh HA, Hashem R, Forbes A, Dilwayo TR, Duaso M, Sturt $\mathrm{J}$, et al. A systematic review of ultrasound-detected lipohypertrophy in insulin-exposed people with diabetes. Diabetes Therapy. 2018;9(5):1741-56.

3. Baruah MP, Kalra S, Bose S, Deka J. An audit of insulin usage and insulin injection practices in a large Indian cohort. Indian $\mathbf{J}$ Endocrinol Metab. 2017;21(3):443-52.

4. Kalra S, Mithal A, Sahay R, John M, Unnikrishnan A, Saboo B, et al. Indian injection technique study: injecting complications, education, and the health care professional. Diabetes Therapy. 2017;8(3):659-72.
5. Barola A, Tiwari P, Bhansali A, Grover S, Dayal D. Insulin-related lipohypertrophy: lipogenic action or tissue trauma? Front Endocrinol. 2018;9:638.

6. Gupta SS, Gupta KS, Gathe SS, Bamrah P, Gupta SS. Clinical implications of lipohypertrophy among people with type 1 diabetes in India. Diabetes Technol Ther. 2018;20(7):483-91.

7. Sürücü HA, Arslan HO. Lipohypertrophy in individuals with type 2 diabetes: prevalence and risk factors. J Caring Sci. 2018;7(2):6774.

8. Mo'men Al Ajlouni MA, Batieha A, Ajlouni K. Prevalence of lipohypertrophy and associated risk factors in insulin-treated patients with type 2 diabetes mellitus. Int J Endocrinol Metab. 2015;13(2):e20776.

9. Al Hayek AA, Robert AA, Braham RB, Al Dawish MA. Frequency of lipohypertrophy and associated risk factors in young patients with type 1 diabetes: a cross-sectional study. Diabetes Therapy. 2016;7(2):259-67.

10. Deeb A, Abdelrahman L, Tomy M, Suliman S, Akle M, Smith M, et al. Impact of insulin injection and infusion routines on Lipohypertrophy and glycemic control in children and adults with diabetes. Diabetes Therapy. 2019;10(1):259-67.

11. Pahuja V, Punjot P, Fernandes G, Chatterjee N. Exploring the factors associated with lipohypertrophy in insulin-treated type 2 diabetes patients in a tertiary care hospital in Mumbai, India. Int $\mathrm{J}$ Diabetes Dev Ctries. 2019; https://doi.org/10.1007/s13410-01900735-0.

12. Campinos C, Le Floch J-P, Petit C, Penfornis A, Winiszewski P, Bordier L, et al. An effective intervention for diabetic lipohypertrophy: results of a randomized, controlled, prospective multicenter study in France. Diabetes Technol Ther. 2017;19(11): 623-32.

13. Tandon N, Kalra S, Balhara YS, Baruah MP, Chadha M, Chandalia $\mathrm{HB}$, et al. Forum for Injection Technique (FIT), India: The Indian recommendations 2.0, for best practice in Insulin Injection Technique, 2015. Indian J Endocr Metab. 2015;19:317-31.

Publisher's note Springer Nature remains neutral with regard to jurisdictional claims in published maps and institutional affiliations. 